Please do not remove this page

RMIT

UNIVERSITY

\title{
Gold Nanoparticle Biodistribution and Toxicity: Role of Biological Corona in Relation with Nanoparticle Characteristics
}

Carnovale, Catherine; Bryant, Gary; Shukla, Ravi; Bansal, Vipul

https://researchrepository.rmit.edu.au/esploro/outputs/9921863261001341/filesAndLinks?institution=61RMIT_INST\&index=null

Carnovale, C., Bryant, G., Shukla, R., \& Bansal, V. (2017). Gold Nanoparticle Biodistribution and Toxicity: Role of Biological Corona in Relation with Nanoparticle Characteristics. In Metal Nanoparticles in Pharma (pp. 419-436). Springer. https://doi.org/10.1007/978-3-319-63790-7_18

Document Version: Accepted Manuscript

Published Version: https://doi.org/10.1007/978-3-319-63790-7_18

Repository homepage: https://researchrepository.rmit.edu.au

(c) Springer International Publishing AG 2017

Downloaded On 2023/04/27 01:15:54 +1000 


\title{
Role of biological corona formed on gold nanoparticle surface on their uptake and toxicity
}

\author{
Catherine Carnovale, ${ }^{1,2}$ Gary Bryant, ${ }^{2}$ Ravi Shukla ${ }^{1}$ and Vipul Bansal ${ }^{1 *}$ \\ ${ }^{1}$ Ian Potter NanoBioSensing Facility, NanoBiotechnology Research Laboratory School of Science, RMIT \\ University, GPO Box 2476, Melbourne, VIC 3001, Australia \\ ${ }^{2}$ Centre for Molecular and Nanoscale Physics, School of Science, RMIT University, GPO Box 2476, \\ Melbourne, VIC 3001, Australia \\ *E-mail: vipul.bansal@rmit.edu.au, Phone: +61 399252121
}

\begin{abstract}
It has long been acknowledged that parameters such as nanoparticle size, shape and surface charge play distinct roles in the way nanomaterials interact with their surrounding biological environment. However, the importance of a nanoparticle's biological corona and the magnitude of its effect have become subject to recent attention. For the purpose of this Chapter, we intend to consider the biological corona as the layer of organic molecules, typically proteins, derived from biological systems that bind to nanoparticle surfaces when nanoparticles are exposed to a biological environment. The protein corona has profound implications on cellular uptake and toxicity of AuNPs, though a deeper understanding of the properties that govern corona formation would provide a greater opportunity for scientists to create tailored AuNPs for specific applications. Specifically, the information provided in this Chapter predominantly deals with gold nanoparticles (AuNPs); however these principles are likely to be extended to other nanomaterials including silver, and other metal oxide nanoparticles.
\end{abstract}

KEYWORDS: Gold nanoparticle, protein corona, cellular uptake, cellular toxicity, size, shape.

\section{Introduction}

It is thought that the protein corona confers a nanoparticle its biological identity, whereby cellular interactions take place based on the abundance and conformation of proteins bound on the particle's surface, rather than the bare surface itself (Alkilany et al. 2009; Lundqvist et al. 2004). However the properties of the underlying AuNP - that is, its size, shape, surface charge and subsequent functionalisation are thought to have bearing on the eventual corona that forms (Fig 1) 
(Casals et al. 2011; Rahman et al. 2013). Using this knowledge, it may be possible to design AuNP based therapies which target specific cell types, cellular locations, or indeed, even evade cellular uptake by linking these material properties with coronal formation behavior. This Chapter therefore attempts to correlate the effect of modifications in AuNP size, shape and surface characteristics with protein corona formation.

The so-called protein corona is the term given to the protein rich coating that is formed at the nanoparticle surface, during a process which commences almost immediately upon the introduction of inorganic materials such as AuNPs to biological fluids (Casals et al. 2010a; Lynch et al. 2007; Mahmoudi et al. 2011). The immediate phase, which is often invoked within seconds, involves the creation of a soft corona (Fig 2). During this initial period, highly mobile proteins within the surrounding environment decorate the surface of the particle. Over a longer time scale, via means of Vroman's effect these initial proteins are displaced by others (Ehrenberg et al. 2009). In what is deemed the hard corona, proteins which possess greater affinity for the surface of the particle replace the loosely bound species to form a more stable complex.

This kinetic process ensures that the nature of the corona will transition over time, with respect to the species bound and their relative abundance on the surface. While initially several hundred protein species may compete for a place on the particle's surface as part of the soft corona, it is thought that the hard corona has a more simplified composition, comprising of approximately 10-50 different proteins (Mahmoudi et al. 2011). Most commonly, these proteins include albumin, immunoglobulin G, fibrinogen and apolipoproteins due to their abundance in the blood. However, it should be noted that their concentration within the blood does not necessarily draw a parallel to their relative concentration within the corona (Rahman et al. 2013).

To observe the interplay between such proteins and nanomaterials in vitro, models employing bovine serum albumin (BSA) or human serum albumin (HSA) are often used (Boulos et al. 2013; Khan et al. 2015; Lacerda et al. 2010; Sen et al. 2011). Both systems allow observation of the interactions which occur between nanomaterials and serum albumin, which is normally produced by the liver and makes up approximately half of the total serum proteins found in vivo. This more simplified system allows researchers to create predictions for the behavior of AuNPs at the biological interface. Due to the complexities of these interactions, the vast majority of studies carried out to date have been on simplified in vitro systems, while in vivo studies in this area are notoriously difficult to interpret and relatively scarce (Hadjidemetriou et al. 2017).

While it is logical to expect that changes in the physical nature of the particles will affect the way in which proteins interact with AuNPs at the nanoscale, one of the most pertinent questions arises on how this knowledge could be exploited for the development of AuNP based therapeutic and diagnostic tools. While therapies incorporating AuNPs 
may take many forms, some of these therapies may require extended circulation time for optimal efficacy and must therefore be designed to avoid initiation of an immune response (Aggarwal et al. 2009; von Maltzahn et al. 2009). Such a response would eventuate in the capture of AuNPs by specific systems which operate to remove foreign material from the body, such as the reticuloendothelial system (RES) or the mononuclear phagocyte system (MPS) (Jones et al. 2013; Mosqueira et al. 1999; Pastorino et al. 2007). Specific proteins called opsonins circulate within the blood to act as signalers for the immune system and upon locating an AuNP, their resultant binding - known as opsonisation may result in clearance of the particles from the body (R Gref et al. 2000; Jones et al. 2013; Moghimi et al. 2001; Soppimath et al. 2001). Known opsonins include but are not limited to immunoglobulins (IgG and $\operatorname{IgM}$ ) as well as specific complement proteins, such as, C3, thrombospondin and fibrinogen. Naturally the human body is capable of an equal and opposing force, known as disopsonisation, whereby specific proteins are capable of having the opposite effect. An example of such a protein is albumin, however one of the most commonly used artificial disopsonin is polyethylene glycol (PEG). Employed by researchers for decades to improve biocompatibility, coating an object in PEG macromolecules or PEGylation makes it possible to decrease the extent of protein adsorption thereby diminishing the risk of opsonisation (Jokerst et al. 2011; Jones et al. 2013; Romberg et al. 2008).

Through a combination of well-known practices and newly emerging research related to the effect of various AuNP traits on protein corona formation, researchers will be able to optimally craft AuNP based therapies which carry the characteristics necessary for their success. Said relationships are invariably complicated and as such a critical review of the current state of research is necessary to establish the current level of understanding in this area.

\section{Establishing a relationship between gold nanoparticle size and protein corona formation}

Generally speaking, an observable difference has been noted in the protein binding behavior of AuNPs of different sizes. More specifically however, changes in the degree of curvature present on the surface of spherical AuNPs are thought to be responsible for the differences observed (Benetti et al. 2013; Casals et al. 2010a; Chithrani et al. 2007; Klein 2007; Lundqvist et al. 2004). When considering spherically-shaped particles, the concept of size can be thought of not only as an increase in the particle diameter as the particle gets larger, but also a decrease in surface curvature. This effect can be examined by comparing the binding which occurs on the surface of spherical AuNPs with the binding of proteins onto flat gold surfaces. At this point, it may again be noted that such size dependent effects appear to be relevant to all nanoparticles, regardless of their elemental composition.

As an evidence of this point, Chah et al. studied the binding of yeast iso-1-cytochrome c (Cyt c) on to the surface of 19 $\mathrm{nm}$ AuNPs as well as flat Au films to monitor the conformational changes that proteins undergo upon binding to various $\mathrm{Au}$ surfaces (Chah et al. 2005). While the group observed Cyt $\mathrm{c}$ undergoing reversible conformational changes after 
binding to the flat Au supports, the same was not observed after binding to the surface of the spherical AuNPs. While the exact reason behind this difference was not established, it can be understood that differences in the geometry of the Au surfaces may either permit or prohibit certain proteins from binding, depending on their own three dimensional constraints (Karajanagi et al. 2004; Lundqvist et al. 2004; Roach et al. 2006). Furthermore, upon binding, the amount of surface curvature may dictate the degree to which the protein is able to contact and effectively bind to the particle surface. This determines not only the stability of the protein in its bound position but also has implications on the protein functionality (Fig 3). In the case of Cyt c, it appears that the binding of the protein to surfaces of a particular curvature may diminish protein functionality by inducing conformational or structural changes.

In an effort to understand the implication of AuNP size on the species of proteins which bind, Dobrovolskaia et al. incubated citrate stabilized 30 and $50 \mathrm{~nm}$ AuNPs with plasma proteins (Dobrovolskaia et al. 2009). While it was difficult to discern the size of the particle which bound a greater amount of protein (even after normalizing the dose of the particles for surface area), the findings from this study indicate that $30 \mathrm{~nm}$ AuNPs bound a larger range of protein species. This study identified 48 individual proteins on the surface of the $30 \mathrm{~nm}$ AuNPs as compared to 21 proteins on the $50 \mathrm{~nm}$ particles. Furthermore, despite fibrinogen being found to be the most abundant protein on both AuNPs, there were only 14 proteins common to both particles. These findings indicate that certain proteins may preferentially bind to particles due to their size or surface curvature which will, in turn, have implications on the kinetics, distribution and clearance of the particles within and from the bloodstream in vivo.

Due to their antimicrobial activity, the use of silver nanoparticles in coating materials for implantable medical devices is the subject of much research (Davoudi et al. 2014; Sondi et al. 2004). Despite this, Durán et al. have noted a significant gap in the literature, whereby the interaction of silver nanoparticles with plasma proteins has gone relatively unreported (Durán et al. 2015). The review highlights the need for further investigation in this area by discussing an interesting study which showed key differences in the interaction of normal, and cancerous human plasma with silver nanoparticles. In the study performed by Feng et al. using surface-enhanced Raman spectroscopy, the group were able to distinguish the behavior of cervical cancer plasma proteins by measuring the intensity of the SERS peak after incubation with silver nanoparticles (Feng et al. 2013). The peak - which is generated from the amide I band of HSA - was found to be more intense due to increased expression in the relative amount of proteins in the $\alpha$-helix conformation which points out that specific protein corona variations can be detected in a cancerous environment. For further discussion on the relationship between silver nanoparticles and the evolution of the protein corona, we refer the reader to Durán et al. 2015.

This idea of a personalized protein corona, which varies depending on the pathology of the patient was reviewed by Corbo et al. highlighting the unique protein biomarkers that accompany common diseases such as cancer, inflammation 
and diabetes (Corbo et al. 2017). The clinical relevance of this occurrence is yet to be exploited which the group relates to the difficulty of understanding nano-bio interactions.

In another study utilizing similar citrate stabilized AuNPs, but of sizes ranging from 4 - $40 \mathrm{~nm}$, Casals et al. probed the effect of size on the timing of the evolution of the protein corona in cell culture conditions, i.e., cell culture media supplemented with fetal bovine serum (Casals et al. 2010a). The group found particles of 10-40 nm forming an albumin rich protein corona, while the smallest particles $(4 \mathrm{~nm})$ did not form a corona, despite extended incubation in serum protein. A high concentration of albumin bound on the surface of AuNPs may indicate that the particles would likely avoid initiating an immune response if used in vivo, however further work would be required to substantiate this. Furthermore, interesting differences were observed between the stability of the protein coronas which formed on the 10 and $40 \mathrm{~nm}$ AuNPs. While the $10 \mathrm{~nm}$ AuNPs were capable of forming an initial soft corona, which transitioned to permanent hard corona over time; the $40 \mathrm{~nm}$ particles possessed a hard corona that was by comparison both less dense and less persistent. With this knowledge, this study inferred that the $10 \mathrm{~nm}$ AuNPs were capable of generating the optimal protein coverage for evasion of the immune system and might be the ideal size for AuNPs based therapies which require this characteristic.

Encompassing a wider size range of citrate stabilized AuNPs ranging from 5-100 nm, Lacerda and coworkers investigated similar AuNP-protein interactions (Lacerda et al. 2010). While the group observed binding of blood plasma proteins to all sizes examined, the trends indicated stronger binding and a thicker coating of proteins with increasing AuNP size (Fig 4). The trend was shown to be size dependent up to approximately $50 \mathrm{~nm}$ after which, saturation was encountered. The group reasoned that the larger AuNPs were able to more efficiently organize and 'pack' proteins bound to the surface. Conversely the cooperativity of binding, i.e., the affinity of a particular protein for a nanoparticle after the binding of successive proteins, was shown to decrease with AuNP size in most cases.

Seeking to develop AuNPs with optimal properties for in vivo applications, Walkey et al., explored the relationship between AuNP size and PEG grafting density (Walkey et al. 2012). The group synthesized citrate stabilized AuNPs of 15-90 nm size and subsequently grafted $5 \mathrm{kDa}$ PEG molecules with grafting densities ranging from 0 (ungrafted) to 1.25 PEG/nm². While the ability of PEG to assist in evasion of the MPS is well documented, the specific parameters that control this effect are not fully understood. Macrophage uptake could not be completely avoided, however the grafting density of PEG and AuNP size were found to impact on the degree of AuNPs uptake by macrophages. As expected, high density PEG grafting was more effective in blocking macrophage uptake due to blockage of serum proteins absorption on to the AuNP surface. Additionally, decreasing AuNP size, and therefore increasing curvature on the particles surface led to an increase in the amount of serum proteins adsorbed. It was speculated by the authors that 
through controlling these two parameters, the degree of macrophage uptake may be potentially manipulated for optimal therapeutic gain.

While the majority of AuNP size dependent studies have been performed on spherical AuNPs, a small body of work exists relating to rod shaped AuNPs. By changing the size, or more specifically the aspect ratio of the gold nanorods (AuNRs), Chithrani and Chan examined the effect of multiple parameters on cellular uptake and clearance - taking note of the role of the protein corona in this environment (Chithrani et al. 2007). By synthesizing transferrin coated AuNRs of aspect ratios 1 (spherical), 1.5, 3.5 and 6, the group noted a reduced rate of AuNPs uptake with increasing aspect ratio. This trend can potentially be related back to the differences in protein binding behavior which occur on AuNRs of different aspect ratios. This study found that rods with a lower aspect ratio displayed a higher degree of protein binding when compared to rods with higher aspect ratios. Returning to the idea of curvature playing a strong role in protein binding activity, in this case, the increased curvature found on the extremities of low aspect ratio rods was hypothesized to be responsible for the trend. Furthermore, this study noted that a higher degree of curvature in this case leads to more strongly bound surface proteins.

While there has been a great volume of research activity around AuNPs, we are currently unable to say if results obtained using a specific cell line are predictive of the behavior of the same AuNP with another cell type. Recently, Cheng and coworkers explored the difference in behavior of phagocytic and non-phagocytic cells interacting with different sized PEG coated spherical AuNPs $(5-50 \mathrm{~nm}$ ) (Cheng et al. 2015). This study noted that particles of $50 \mathrm{~nm}$ are preferred candidates for cellular uptake. By performing experiments in the absence and presence of serum proteins, a commonly documented trend showing decreased levels of particle uptake in the presence of serum, was observed. However, this trend was found to be both size and cell type dependent; being more pronounced for larger particles, and in phagocytic cells (Fig 5).

These results illustrate one of the greatest challenges faced by researchers working on in-vitro testing of AuNPs and indeed of all nanomaterials in general. The difficult nature of grouping results to extrapolate the knowledge we currently possess and apply it in varied conditions dictates that each material be tested individually, and in a variety of settings. It is easy to see that this approach is both labor and time intensive. In the attempt to relate AuNP size and protein corona formation general trends have been observed, most evidently the ability of AuNPs with increased curvature to accommodate proteins with greater ease on their surface.

Establishing a relationship between gold nanoparticle shape and protein corona formation 
While arguably holding equal importance to the effect of size, the effect of shape on the evolution of a protein corona around AuNPs is less understood. Spherical nanoparticles are often seen by researchers as a logical starting point for biological studies and thus, there are markedly less number of studies performed on AuNPs of differing shape. Furthermore, synthesizing AuNPs of varying shape brings an additional layer of complexity to the synthesis process, particularly when doing comparative studies. For the observation of true shape effects, parameters such as chemical structure, surface charge and to an extent, surface area must be kept constant. In this way, only systematic studies which keep these factors constant while solely varying the particle shape can be treated as true indicators of the effect of AuNP shape on protein corona formation. From the small pool of studies conducted, it has been observed that the geometric properties that determine shape such as curved surfaces, planar sides, sharp edges, corners, ridges and pores affect the way in which proteins interact with a given particle (Fig 6). The relationship does not appear to be governed by general rules but rather by the specific nature of each protein and nanoparticle, respectively. In this way, geometric features have been described as exhibiting both blocking and assistive effects for the binding of proteins depending on protein size and conformation (Mahmoudi et al. 2011).

Due, in part to the difficulty of performing highly precise systematic studies in vitro, in silico simulations are utilized as a predictive tool to observe the way in which different shaped AuNPs and proteins may interact. Ramezani and coworkers performed a dynamic simulation study to probe the interaction of HSA binding to cubic and spherical AuNPs (Ramezani et al. 2014). The study maintained the common idea that curved surfaces such as those found on spherical AuNPs better accommodate the binding of HSA molecules. Furthermore, this study calculated the distance which would theoretically separate the protein molecule from the AuNPs should they interact. These calculations determined that HSA molecules are indeed able to position closer to the surface of a spherical particle when compared to the cubic particle. However despite the increased distance, the planar surfaces which confine the cubic particle cause the albumin to unfold, generating structural changes much more strongly than spherical AuNPs. These results highlight the possibility that shape can elicit effects on secondary protein structures and may therefore be capable of altering the activity of proteins as they attempt to bind to the particles surface.

Carefully controlled in vitro experiments such as those by Gagner and coworkers, examined the effect of shape on binding with two specific enzymes - lysozyme and $\alpha$-chymotrypsin (Gagner et al. 2011). The study attempted to facilitate comparability between the synthesized particles (rods and spheres) by performing surface functionalisation to provide similar surfaces to both shapes, despite variations which occurred during the synthesis. Lysozyme showed a minor loss of secondary structure upon binding to both particles, which subsequently caused particle aggregation and led to a major loss of activity. While $\alpha$-chymotrypsin was able to preserve its structure after interaction with both particles at low concentrations, at higher surface densities where a monolayer of protein was formed on AuNRs, this 
resulted in significant secondary structure disruption and loss of activity. However, if conditions permitted the development of multiple layers of coverage over the AuNR surface, $\alpha$-chymotrypsin recovered its original activity level. Such results strongly indicate the effects that different shapes may exert on protein structure and function, however further work is required to understand the rules that govern these interactions. Overall it was noted that both proteins achieved higher binding densities on the surface of AuNRs as compared to AuNSs, which the authors concluded was due to the long cylindrical surface, which is comparatively flatter than that of the AuNS and accommodated a higher number of proteins with greater ease.

Adding an additional layer of complexity to this story, in publications first from Caswell et al.(Caswell et al. 2003), and then also Chang et al.(Chang et al. 2005), a contrasting effect was reported. In both cases the groups utilized AuNRs synthesized using CTAB for which it can be assumed that a residual bilayer of CTAB remains on the surface of the AuNRs, preventing agglomeration. Given Gagners hypothesis, we would expect preferential binding of proteins to the flat cylindrical surface of the rods, however in the case of AuNRs synthesized with CTAB, the CTAB molecules appear to tightly pack along these surfaces, preventing subsequent molecules from binding to this surface. Thus, both groups found that proteins preferentially bound to the ends of the rods, where it is thought that CTAB is unable to bind at a high density.

While there is undoubtedly more weight to support the notion of AuNP shape exerting some effect on protein adsorption, it is possible that the effect is less pronounced or may not exist at all in some instances. This is evident from the research performed by Boulos et al, that examined the way BSA binds to AuNPs of different shapes (Boulos et al. 2013). The team synthesized AuNRs and AuNSs, surface functionalizing both shapes such that they were either positively charged, or negatively charged, or neutral. While many surface charge specific effects were observed, this study reportedly noted no difference which could be related to the shape of the particles. Similarly, Mirsadeghi et al. observed no shape dependent effects in protein corona formation around CTAB stabilized AuNRs and AuNSs (Mirsadeghi et al. 2015). Thus it is understood that without the knowledge of the underlying mechanisms that determine these interactions, case by case investigations must be carried out. It is hoped that in this way the factors that dominate these effects will become more apparent as the pool of research grows.

\section{Establishing a relationship between gold nanoparticle surface characteristics and protein corona formation.}

While all AuNPs possess commonality in context of sharing an inner gold core, the degree to which they may differ on the surface remains significant (Fig 7). Surface coating and functionalization steps are commonly employed post synthesis to influence biodistribution, alter circulation time, or increase biocompatibility (R Gref et al. 2000; Rahman et al. 2013). When considering that it is the outermost surface of an AuNP that encounters the biological interface 
initially, it is unsurprising that these characteristics are thought to impact on the formation and composition of the protein corona.

It is in this area of AuNP design that researchers may have great influence on the fate of an AuNP after it enters the body. Research has long shown that without steps being taken to modify the surface of nanoparticle prior to its administration in the blood stream, the immune system will rapidly launch a response to clear and eliminate the introduced particles (Ruxandra Gref et al. 1994). With this knowledge, a number of studies have been performed, not only to create surface coatings highly specific for the particle's intended use, but also to gain knowledge on the general principles that govern AuNP surface-protein corona interactions.

Casals et al. studied the effect of citrate stabilized AuNPs which were modified to be either positively or negatively charged to determine the effect of surface charge on protein corona formation (Casals et al. 2010b). Most interestingly, the particles which were modified with mercaptoundecanoic acid to provide a negatively charged surface were unable to form a hard or persistent protein corona despite prolonged incubation time. This effect was attributed to the repulsion of negatively charged proteins from within the cell culture medium. In contrast, aminoundecanethiol modified, positively charged AuNPs were capable of forming a soft corona which was persistent and highly stable.

The realm of AuNP surface modification is boundless, with modifications of increasing complexity being reported particularly for the development of AuNP based therapies. While many applications of AuNPs will require evasion of the immune system, it can also be understood that certain applications will require increased cellular uptake or prompt clearance from the blood stream. One such study by Chinen and colleagues utilized spherical nucleic acid (SNA) nanoparticles possessing an AuNP core surrounded by a 3' thiol-modified guanine-rich sequence (Chinen et al. 2015). The study determined that the modified structure of the oligonucleotide affected protein corona dramatically in its composition. This altered protein coating was shown to significantly increase the uptake of AuNPs by macrophages.

The impact of protein adsorption on cellular uptake was also probed by Zhu and co-workers who synthesized 14 AuNPs, each possessing the same inner core, but bearing different functional groups to impart a wide spectrum of hydrophobicity levels (Zhu et al. 2012). When tested in the absence of serum, AuNPs had similar uptake levels, however when tested in serum supplemented media, increased hydrophobicity led to decreased cellular uptake (Fig 8). This supports many other studies which show decreased cellular uptake in the presence of protein. The authors reason that BSA binds to the particle surface with increased strength as the hydrophobicity of the particles increase. This presumably creates a repulsion effect between the negative charges present on BSA and the cellular membrane which leads to decreased levels of cellular uptake; however the authors also noted that the effect is also contributed by other proteins within the medium. 
The impact of the composition of the protein corona on biological behavior of nanoparticles has been investigated by several groups; however it must be stated that although changes in composition are reported, we are yet to know how to maximally exploit this effect to create particles that carry a specific composition of proteins. Additionally, the effect on biomolecule structure and activity after incorporation into the protein corona is largely unknown. While RadauerPreiml and co-workers were able to detect modulated immune reactions after conjugation of common allergens to 50 nm AuNPs as compared with exposure to the allergens alone, they are unable to report predictable or constant results in this area of research (Radauer-Preiml et al. 2016). While the authors were able to conclude that allergen inclusion in the protein corona can increase protease activity and therefore change the process of allergen sensitization, numerous unknowns including a high degree of allergic donor variability, made it impossible for the results to be clearly interpreted.

While clarity in this area may be seen in the future, current studies have uncovered a consistent message in the case of PEG modified AuNPs with numerous studies finding a correlation between the molecular weight of the PEG molecule used (or its chain length) and the amount of protein that is bound to the nanoparticle. In this case it is typically noted that using PEG with a high molecular weight leads to a particle which interacts less with proteins and forms a lower density protein coating.

With this knowledge, Walkey et al. examined the difference in the compositions of the protein coronas that developed on the surface of AuNPs with citrate and PEG surface coatings (Walkey et al. 2012). As expected, the citrate stabilized AuNPs demonstrated a higher degree of interaction with plasma proteins (binding a greater number of proteins) as compared with the PEG coated particles (Fig 9). However, the compositional study yielded interesting results with respect to the levels of complement protein $\mathrm{C} 3$ on the surface of the particles. Complement protein $\mathrm{C} 3$ is an opsonin, known to increase macrophage response and the subsequent elimination of material from the bloodstream. In this study, when the bound fraction of complement component C3 protein was measured, it was found to be made up of $>30 \% \mathrm{w} / \mathrm{w}$ of total protein adsorbed onto the citrate AuNPs in contrast to $<5 \% \mathrm{w} / \mathrm{w}$ on PEG modified particles. These results add complexity to the effect of PEGylation, whereby it is unclear if reduced uptake of nanoparticles is due to decreased protein interactions or the formulation of a specific protein coating which results in macrophage evasion. It is possible that the effect observed may be a combination of both actions; however further research is needed to clarify this argument.

The use of PEG as a nanoparticle surfactant is commonly employed for its ability to provide extended and undetected circulation times within the blood, however attachment of such molecules to the nanoparticle surface must be performed covalently, is notably laborious and difficult to control. Recently Muller et al. devised a novel alternative to coat the 
surface of nanoparticles with biodegradable polymeric surfactants derived from poly(-phosphoester) in a non-covalent attachment manner. The surface coating - which can be applied to nanoparticles of any species - carries the ability to be easily tuned in terms of length and hydrophobicity to create nanoparticles with tailored surfaces to suit their specific purpose (Müller et al. 2017).

While the vast majority of protein corona studies are designed to investigate the behavior of AuNPs at the nano-blood interface, Mahmoud and co-workers investigated protein corona effects at the nano-skin interface (Mahmoud et al. 2016). By using excised human skin samples, the group was able to investigate the behavior of AuNRs with particular respect to stability by using particles of different surface charges (positive, negative and neutral). Findings suggested that positively charge AuNRs interacted with proteins released from the inner layers (dermis) to form a protein corona which resulted in rapid particle agglomeration. The lack of interaction between negatively charged proteins and biomolecules allowed the neutral and negatively charged AuNRs to maintain their stability when in contact with the skin for up to 24 hours. This outcome has large implications for the development of dermal based nanotherapeutics, particularly for therapies that require the preservation of AuNRs characteristic absorption spectrum, which was lost in the case of the cationic particles after agglomeration.

\section{Conclusion}

It must be considered that research relating to the evolution and influence of the protein corona on AuNP uptake and toxicity is a relatively new field, and as such, the area is continually advancing. While we are yet to know exactly which parameters lead in governing AuNP-protein interactions, certain trends are beginning to emerge from the research with an increasing clarity. Over time, it is hoped that these trends will solidify to become a guide for researchers developing novel AuNP based therapies. In this way, it will be possible to synthesize AuNPs possessing highly specific characteristics which predetermine their toxic effects, uptake constraints and biodistribution in vivo. With work in this popular field of research continuing, an increase in the commercialization of AuNP based therapies is forecast to be the likely outcome of such progress.

\section{Figures}

Figure 1: Different physico-chemical properties of gold nanoparticles (AuNPs) that may influence their interaction with proteins. Reprinted with permission from (Carnovale et al. 2016) Progress in Materials Science.

Figure 2: Schematic depicting the progressive formation of the soft and hard coronas. Reprinted with permission from (Wolfram et al. 2014) Colloids and Surfaces B: Biointerfaces 
Figure 3: Schematic representation of the likely outcomes that affect protein structure and function which may occur after binding to an AuNP surface. Reprinted with permission from (Saptarshi et al. 2013). Licensed under CC BY 2.0.

Figure 4: Graphical representation of the trends reported by Lacerda et al. showing stronger binding and a thicker coating of proteins developing on AuNPs with increasing size. Adapted with permission from (Lacerda et al. 2010). Copyright 2009 American Chemical Society.

Figure 5: Results obtained by Cheng et al. showing decreased particle uptake in the presence of serum supplemented media (c-DMEM) in (a) phagocytic cells and (b) non phagocytic cells. Adapted with permission from (Cheng et al. 2015). Copyright 2015 American Chemical Society.

Figure 6: Representation of a molecular simulation performed by Li et al. showing protein binding of different shaped AuNPs. Reprinted with permission from (Li et al. 2015). Copyright Royal Society of Chemistry.

Figure 7: Depiction of the multiple surface functionalisation options available for altering the surface of AuNPs. Reprinted with permission from (Conde et al. 2014). Licensed under CC BY 2014.

Figure 8: Results obtained by Zhu et al. showing the correlation between increased hydrophobicity and decreased cellular uptake arising in the presence of serum. The properties of AuNP 1-4 are consistent but for increasing alkane chain lengths to confer varying degrees of hydrophobicity. Reprinted with permission from (Zhu et al. 2012). Copyright Wiley-VCH.

Figure 9: Schematic representing the effect of PEG density on serum protein adsorption to AuNPs. As demonstrated by Walkey et al., AuNPs with increasing PEG density show reduced protein binding. Adapted with permission from (Walkey et al. 2012). Copyright 2012 American Chemical Society.

\section{References:}

Aggarwal P, Hall J B, McLeland C B, Dobrovolskaia M A, \& McNeil S E (2009) Nanoparticle interaction with plasma proteins as it relates to particle biodistribution, biocompatibility and therapeutic efficacy. Advanced drug delivery reviews 61: 428-437 
Alkilany A M, Nagaria P K, Hexel C R, Shaw T J, Murphy C J, \& Wyatt M D (2009) Cellular Uptake and Cytotoxicity of Gold Nanorods: Molecular Origin of Cytotoxicity and Surface Effects. Small 5: 701-708

Benetti F, Fedel M, Minati L, Speranza G, \& Migliaresi C (2013) Gold nanoparticles: role of size and surface chemistry on blood protein adsorption. Journal of Nanoparticle Research 15: 1-9

Boulos S P, Davis T A, Yang J A, Lohse S E, Alkilany A M, Holland L A, \& Murphy C J (2013) Nanoparticle-Protein Interactions: A Thermodynamic and Kinetic Study of the Adsorption of Bovine Serum Albumin to Gold Nanoparticle Surfaces. Langmuir 29: 14984-14996

Carnovale C, Bryant G, Shukla R, \& Bansal V (2016) Size, shape and surface chemistry of nano-gold dictate its cellular interactions, uptake and toxicity. Progress in Materials Science 83: 152-190

Casals E, Pfaller T, Duschl A, Oostingh G J, \& Puntes V (2010a) Time evolution of the nanoparticle protein corona. Acs Nano 4

Casals E, Pfaller T, Duschl A, Oostingh G J, \& Puntes V (2010b) Time Evolution of the Nanoparticle Protein Corona. Acs Nano 4: 3623-3632

Casals E, Pfaller T, Duschl A, Oostingh G J, \& Puntes V F (2011) Hardening of the NanoparticleProtein Corona in Metal ( $\mathrm{Au}, \mathrm{Ag}$ ) and Oxide (Fe3O4, $\mathrm{CoO}$, and $\mathrm{CeO} 2)$ Nanoparticles. Small 7: $3479-3486$

Caswell K, Wilson J N, Bunz U H, \& Murphy C J (2003) Preferential end-to-end assembly of gold nanorods by biotin-streptavidin connectors. Journal of the American Chemical Society 125 : 13914-13915

Chah S, Hammond M R, \& Zare R N (2005) Gold Nanoparticles as a Colorimetric Sensor for Protein Conformational Changes. Chemistry \& Biology 12: 323-328

Chang J-Y, Wu H, Chen H, Ling Y-C, \& Tan W (2005) Oriented assembly of Au nanorods using biorecognition system. Chem. Commun.: 1092-1094

Cheng X, Tian X, Wu A, Li J, Tian J, Chong Y, . . Ge C (2015) Protein Corona Influences Cellular Uptake of Gold Nanoparticles by Phagocytic and Nonphagocytic Cells in a Size-Dependent Manner. ACS Applied Materials \& Interfaces 7: 20568-20575

Chinen A B, Guan C M, \& Mirkin C A (2015) Spherical Nucleic Acid Nanoparticle Conjugates Enhance G-Quadruplex Formation and Increase Serum Protein Interactions. Angewandte Chemie International Edition 54: 527-531 
Chithrani B D, \& Chan W C W (2007) Elucidating the mechanism of cellular uptake and removal of protein-coated gold nanoparticles of different sizes and shapes. Nano Letters 7: 1542-1550

Conde J, Dias J T, Grazú V, Moros M, Baptista P V, \& de la Fuente J M (2014) Revisiting 30 years of biofunctionalization and surface chemistry of inorganic nanoparticles for nanomedicine. Frontiers in chemistry 2: 48

Corbo C, Molinaro R, Tabatabaei M, Farokhzad O C, \& Mahmoudi M (2017) Personalized protein corona on nanoparticles and its clinical implications. Biomaterials Science 5: 378-387

Davoudi Z M, Kandjani A E, Bhatt A I, Kyratzis I L, O'Mullane A P, \& Bansal V (2014) Hybrid antibacterial fabrics with extremely high aspect ratio $\mathrm{Ag} / \mathrm{AgTCNQ}$ nanowires. Advanced Functional Materials 24: 1047-1053

Dobrovolskaia M A, Patri A K, Zheng J, Clogston J D, Ayub N, Aggarwal P, ... McNeil S E (2009) Interaction of colloidal gold nanoparticles with human blood: effects on particle size and analysis of plasma protein binding profiles. Nanomedicine : nanotechnology, biology, and medicine 5: 106-117

Durán N, Silveira C P, Durán M, \& Martinez D S T (2015) Silver nanoparticle protein corona and toxicity: a mini-review. Journal of nanobiotechnology 13: 55

Ehrenberg M S, Friedman A E, Finkelstein J N, Oberdorster G, \& McGrath J L (2009) The influence of protein adsorption on nanoparticle association with cultured endothelial cells. Biomaterials 30: $603-610$

Feng S, Lin D, Lin J, Li B, Huang Z, Chen G, ... Zeng H (2013) Blood plasma surface-enhanced Raman spectroscopy for non-invasive optical detection of cervical cancer. Analyst 138: 39673974

Gagner J E, Lopez M D, Dordick J S, \& Siegel R W (2011) Effect of gold nanoparticle morphology on adsorbed protein structure and function. Biomaterials 32: 7241-7252

Gref R, Lück M, Quellec P, Marchand M, Dellacherie E, Harnisch S, . . Müller R (2000) 'Stealth' corona-core nanoparticles surface modified by polyethylene glycol (PEG): influences of the corona (PEG chain length and surface density) and of the core composition on phagocytic uptake and plasma protein adsorption. Colloids and Surfaces B: Biointerfaces 18: 301-313

Gref R, Minamitake Y, Peracchia M T, Trubetskoy V, Torchilin V, \& Langer R (1994) Biodegradable long-circulating polymeric nanospheres. Science 263: 1600-1603

Hadjidemetriou M, \& Kostarelos K (2017) Nanomedicine: Evolution of the nanoparticle corona. Nature Nanotechnology 12: 288-290 
Jokerst J V, Lobovkina T, Zare R N, \& Gambhir S S (2011) Nanoparticle PEGylation for imaging and therapy. Nanomedicine 6: 715-728

Jones S W, Roberts R A, Robbins G R, Perry J L, Kai M P, Chen K, . . DeSimone J M (2013) Nanoparticle clearance is governed by Th1/Th2 immunity and strain background. The Journal of clinical investigation 123: 3061-3073

Karajanagi S S, Vertegel A A, Kane R S, \& Dordick J S (2004) Structure and function of enzymes adsorbed onto single-walled carbon nanotubes. Langmuir 20: 11594-11599

Khan S, Gupta A, Verma N, \& Nandi C (2015) Kinetics of protein adsorption on gold nanoparticle with variable protein structure and nanoparticle size. The Journal of chemical physics 143: 164709

Klein J (2007) Probing the interactions of proteins and nanoparticles. Proceedings of the National Academy of Sciences 104: 2029-2030

Lacerda S H, Park J J, Meuse C, Pristinski D, Becker M L, Karim A, \& Douglas J F (2010) Interaction of gold nanoparticles with common human blood proteins. ACS Nano 4: 365-379

Li Y, Kröger M, \& Liu W K (2015) Shape effect in cellular uptake of pegylated nanoparticles: comparison between sphere, rod, cube and disk. Nanoscale 7: 16631-16646

Lundqvist M, Sethson I, \& Jonsson B-H (2004) Protein adsorption onto silica nanoparticles: conformational changes depend on the particles' curvature and the protein stability. Langmuir 20: $10639-10647$

Lynch I, Cedervall T, Lundqvist M, Cabaleiro-Lago C, Linse S, \& Dawson K A (2007) The nanoparticle - protein complex as a biological entity; a complex fluids and surface science challenge for the 21st century. Advances in Colloid and Interface Science 134-35: 167-174

Mahmoud N N, Al-Qaoud K M, Al-Bakri A G, Alkilany A M, \& Khalil E A (2016) Colloidal stability of gold nanorod solution upon exposure to excised human skin: Effect of surface chemistry and protein adsorption. The International Journal of Biochemistry \& Cell Biology 75: 223231

Mahmoudi M, Lynch I, Ejtehadi M R, Monopoli M P, Bombelli F B, \& Laurent S (2011) ProteinNanoparticle Interactions: Opportunities and Challenges. Chemical Reviews 111: 5610-5637

Mirsadeghi S, Dinarvand R, Ghahremani M H, Hormozi-Nezhad M R, Mahmoudi Z, Hajipour M J, . . . Mahmoudi M (2015) Protein corona composition of gold nanoparticles/nanorods affects amyloid beta fibrillation process. Nanoscale 7: 5004-5013 
Moghimi S M, Hunter A C, \& Murray J C (2001) Long-circulating and target-specific nanoparticles: theory to practice. Pharmacological reviews 53: 283-318

Mosqueira V C, Legrand P, Gref R, Heurtault B, Appel M, \& Barratt G (1999) Interactions between a macrophage cell line (J774A1) and surface-modified poly (D,L-lactide) nanocapsules bearing poly(ethylene glycol). J Drug Target 7: 65-78

Müller J, Bauer K N, Prozeller D, Simon J, Mailänder V, Wurm F R, . . Landfester K (2017) Coating nanoparticles with tunable surfactants facilitates control over the protein corona. Biomaterials 115: $1-8$

Pastorino F, Marimpietri D, Brignole C, Di Paolo D, Pagnan G, Daga A, . . Ponzoni M (2007) Ligand-targeted liposomal therapies of neuroblastoma. Curr Med Chem 14: 3070-3078

Radauer-Preiml I, Andosch A, Hawranek T, Luetz-Meindl U, Wiederstein M, Horejs-Hoeck J, . . . Duschl A (2016) Nanoparticle-allergen interactions mediate human allergic responses: protein corona characterization and cellular responses. Particle and fibre toxicology 13: 3

Rahman M, Laurent S, Tawil N, Yahia L H, \& Mahmoudi M (2013). Protein-Nanoparticle Interactions: The Bio-Nano Interface: Springer.

Ramezani F, Amanlou M, \& Rafii-Tabar H (2014) Gold nanoparticle shape effects on human serum albumin corona interface: a molecular dynamic study. Journal of Nanoparticle Research 16 : $1-9$

Roach P, Farrar D, \& Perry C C (2006) Surface Tailoring for Controlled Protein Adsorption: Effect of Topography at the Nanometer Scale and Chemistry. Journal of the American Chemical Society 128: 3939-3945

Romberg B, Hennink W E, \& Storm G (2008) Sheddable coatings for long-circulating nanoparticles. Pharmaceutical research 25: 55-71

Saptarshi S R, Duschl A, \& Lopata A L (2013) Interaction of nanoparticles with proteins: relation to bio-reactivity of the nanoparticle. Journal of nanobiotechnology 11: 1-12

Sen T, Mandal S, Haldar S, Chattopadhyay K, \& Patra A (2011) Interaction of gold nanoparticle with human serum albumin (HSA) protein using surface energy transfer. The Journal of Physical Chemistry C 115: 24037-24044

Sondi I, \& Salopek-Sondi B (2004) Silver nanoparticles as antimicrobial agent: a case study on E. coli as a model for Gram-negative bacteria. Journal of colloid and interface science 275: 177-182 
Soppimath K S, Aminabhavi T M, Kulkarni A R, \& Rudzinski W E (2001) Biodegradable polymeric nanoparticles as drug delivery devices. Journal of controlled release 70: 1-20

von Maltzahn G, Park J-H, Agrawal A, Bandaru N K, Das S K, Sailor M J, \& Bhatia S N (2009) Computationally guided photothermal tumor therapy using long-circulating gold nanorod antennas. Cancer research 69: 3892-3900

Walkey C D, Olsen J B, Guo H, Emili A, \& Chan W C (2012) Nanoparticle size and surface chemistry determine serum protein adsorption and macrophage uptake. Journal of the American Chemical Society 134: 2139-2147

Wolfram J, Yang Y, Shen J, Moten A, Chen C, Shen H, .. Zhao Y (2014) The nano-plasma interface: implications of the protein corona. Colloids and Surfaces B: Biointerfaces 124: 1724

Zhu Z J, Posati T, Moyano D F, Tang R, Yan B, Vachet R W, \& Rotello V M (2012) The interplay of monolayer structure and serum protein interactions on the cellular uptake of gold nanoparticles. Small 8: 2659-2663 